\title{
CARLESON MEASURES FOR THE AREA NEVANLINNA SPACES AND APPLICATIONS
}

\author{
BOO RIM CHOE, HYUNGWOON KOO, AND WAYNE SMITH
}

\begin{abstract}
Let $1 \leq p<\infty$ and let $\mu$ be a positive finite Borel measure on the unit disk $D$. The area Nevanlinna-Lebesgue space $N^{p}(\mu)$ consists of all measurable functions $h$ on $D$ such that $\log ^{+}|h| \in L^{p}(\mu)$, and the area Nevanlinna space $N_{\alpha}^{p}$ is the subspace of $N^{p}\left(\left(1-|z|^{2}\right)^{\alpha} d \nu(z)\right)$, where $\alpha>-1$ and $\nu$ is area measure on $D$, consisting of all holomorphic functions. We characterize Carleson measures for $N_{\alpha}^{p}$, defined to be those measures $\mu$ for which $N_{\alpha}^{p} \subset N^{p}(\mu)$. One application is that the spaces $N_{\alpha}^{p}$ are all closed under both differentiation and integration. This is in contrast to the classical Nevanlinna space defined by integration on circles centered at the origin, which is closed under neither. Applications to composition operators and to integral operators are also given.
\end{abstract}

\section{INTRODUCTION}

Let $D$ be the unit disk of the complex plane $\mathbf{C}$ and $H(D)$ be the algebra of all functions holomorphic on $D$. Let $\nu$ be the area measure on $D$ normalized to have total mass 1 . Given $\alpha>-1$, let $\nu_{\alpha}$ be the weighted measure defined by $d \nu_{\alpha}(z)=(\alpha+1)\left(1-|z|^{2}\right)^{\alpha} d \nu(z)$.

For $1 \leq p<\infty$ and $\alpha>-1$, we let $N_{\alpha}^{p}$ denote the space of all functions $f \in H(D)$ such that

$$
\int_{D}\left(\log ^{+}|f|\right)^{p} d \nu_{\alpha}<\infty
$$

We will refer to the spaces $N_{\alpha}^{p}$ as (weighted) area Nevanlinna spaces. Clearly, each area Nevanlinna space is a subalgebra of $H(D)$. The area Nevanlinna spaces are large in the sense that each $N_{\alpha}^{p}$ contains all the well-known Bergman spaces. This follows from standard growth estimates for Bergman functions (see [9, Lemma 3.2]). Also, by the elementary inequalities $\log ^{+} x \leq \log (1+x) \leq \log 2+\log ^{+} x$ for $x \geq 0$, we see that (1.1) holds if and only if $\log (1+|f|) \in L^{p}\left(\nu_{\alpha}\right)$. Next, note that $\log (1+x+y) \leq \log (1+x)+\log (1+y)$ for $x, y \geq 0$. These simple observations lead us to define the "norm" $\|f\|_{N_{\alpha}^{p}}$ of $f \in N_{\alpha}^{p}$ by the formula

$$
\|f\|_{N_{\alpha}^{p}}^{p}=\int_{D}[\log (1+|f|)]^{p} d \nu_{\alpha}
$$

Date: November 16, 2006.

2000 Mathematics Subject Classification. Primary 47B38; Secondary 30H05, 46E10.

Key words and phrases. Carleson measure, Area Nevanlinna space, Composition operator, Volterra operator.

The first two authors were supported by $\operatorname{KOSEF}(\mathrm{R} 01-2003-000-10243-0)$. 
This norm satisfies the triangle inequality and thus induces a translation-invariant metric on $N_{\alpha}^{p}$ defined by $(f, g) \mapsto\|f-g\|_{N_{\alpha}^{p}}$. Also, we have by subharmonicity

$$
\log (1+|f(a)|) \leq C \frac{\|f\|_{N_{\alpha}^{p}}}{(1-|a|)^{(2+\alpha) / p}}, \quad a \in D
$$

for a constant $C=C(p, \alpha)$ and all $f \in N_{\alpha}^{p}$. In particular, this tells us that if $f_{n} \rightarrow f$ in $N_{\alpha}^{p}$, then $f_{n} \rightarrow f$ locally uniformly. Here, the local uniform convergence refers to the uniform convergence on every compact subset of $D$. It follows that each $N_{\alpha}^{p}$ is a translation-invariant complete metric space.

Let $\mu$ be a positive finite Borel measure $\mu$ on $D$ (hereafter we simply write $\mu \geq 0)$. We introduce the spaces $N^{p}(\mu), 1 \leq p<\infty$, which we call area NevanlinnaLebesgue spaces. Namely, we define $N^{p}(\mu)$ to be the space of all measurable functions $h$ on $D$ such that

$$
\|h\|_{N^{p}(\mu)}^{p}:=\int_{D}[\log (1+|h|)]^{p} d \mu<\infty .
$$

This norm also induces a translation-invariant metric on $N^{p}(\mu)$, which is also complete (see [11, Proposition 2.1]). Clearly, $N_{\alpha}^{p}$ is a closed subspace of $N^{p}\left(\nu_{\alpha}\right)$. We also recall the well-known (weighted) Bergman spaces $A_{\alpha}^{p}:=L^{p}\left(\nu_{\alpha}\right) \cap H(D)$, where $\alpha>-1$ and $1 \leq p<\infty$. Note that while the area Nevanlinna(-Lebesgue) spaces are not Banach spaces, the restriction $1 \leq p<\infty$ means that the spaces $A_{\alpha}^{p}$ are. Some of our results assert that certain operators are bounded or compact in a sense that is stronger than the general notion of bounded or compact on a topological vector space. Precise definitions of those strong notions, that we call "metric boundedness" and "metric compactness", are given in Section 2. For now we just mention that on a Banach space these properties coincide with the usual notions of boundedness and compactness.

In what follows, we let $\mathcal{I}$ denote the integration operator defined by

$$
\mathcal{I} f(z)=\int_{0}^{z} f(\zeta) d \zeta, \quad z \in D
$$

for $f \in H(D)$. Also let $\partial=\frac{\partial}{\partial z}$ denote the differentiation operator. We can now state our first result.

Theorem 1.1. Let $1 \leq p<\infty$ and $\alpha>-1$. Given $\mu \geq 0$, the following conditions are equivalent:
(a) $A_{\alpha}^{p} \subset L^{p}(\mu)$;
(b) $N_{\alpha}^{p} \subset N^{p}(\mu)$;
(c) The embedding $N_{\alpha}^{p} \subset N^{p}(\mu)$ is metrically bounded;
(d) $\mathcal{I}\left(N_{\alpha}^{p}\right) \subset N^{p}(\mu)$;
(e) $\mathcal{I}: N_{\alpha}^{p} \rightarrow N^{p}(\mu)$ is metrically bounded;
(f) $\partial\left(N_{\alpha}^{p}\right) \subset N^{p}(\mu)$;
(g) There is a constant $C$ such that

$$
\left\|f^{\prime}\right\|_{N^{p}(\mu)} \leq C\left(1+\|f\|_{N_{\alpha}^{p}}\right)
$$

for $f \in N_{\alpha}^{p}$.

As an immediate consequence of Theorem 1.1 with $\mu=\nu_{\alpha}$, we see (Corollary 3.3) that area Nevanlinna spaces are all closed under differentiation and integration. This is in contrast to differentiation and integration on the classical Nevanlinna 
spaces defined by integration on circles centered at the origin. For $p \geq 1$, a function $f \in H(D)$ is said to belong to $\mathcal{N}^{p}$ if the integrals

$$
\int_{0}^{2 \pi}\left(\log ^{+}\left|f\left(r e^{i \theta}\right)\right|\right)^{p} d \theta
$$

are uniformly bounded for $r<1$. In 1929 A. Bloch and R. Nevanlinna [16] posed the problem of whether $\mathcal{N}^{1}$ is closed under differentiation. Subsequently many counterexamples have been constructed. It is now known that the derivative of a bounded holomorphic function on the unit disk need not belong to $\mathcal{N}^{1}$, so none of the spaces $\mathcal{N}^{p}, p \geq 1$, are closed under differentiation; see for example [6] or [8]. Thus the area Nevanlinna spaces $N_{\alpha}^{p}$ may be viewed as the proper setting for the Bloch-Nevanlinna problem. On the other hand, W. K. Hayman has shown [8] that while the spaces $\mathcal{N}^{p}, p>1$, are closed under integration, the space $\mathcal{N}^{1}$ is not.

Measures $\mu$ that satisfy $A_{\alpha}^{p} \subset L^{p}(\mu)$ have been extensively studied and are known as Carleson measures for $A_{\alpha}^{p}$. We will often call such a measure an $\alpha$-Carleson measure (they are independent of $p$ ). Some basic properties of these measures will be reviewed in Section 2. By analogy we define a Carleson measure for $N_{\alpha}^{p}$ to be a measure $\mu \geq 0$ such that $N_{\alpha}^{p} \subset N^{p}(\mu)$. In this terminology Theorem 1.1 says, in part, that $\mu$ is a Carleson measure for $A_{\alpha}^{p}$ if and only if it is a Carleson measure for $N_{\alpha}^{p}$.

Since the spaces $N_{\alpha}^{p}$ and $N^{p}(\mu)$ are complete translation-invariant metric spaces, it is an immediate consequence of the Closed Graph Theorem that if $\partial\left(N_{\alpha}^{p}\right) \subset$ $N^{p}(\mu)$, then the differentiation operator $\partial: N_{\alpha}^{p} \rightarrow N^{p}(\mu)$ is continuous and hence bounded in the sense of an operator on a topological vector space; see [19, Theorem 1.32]. So we see from Theorem 1.1 that $\partial: N_{\alpha}^{p} \rightarrow N^{p}(\mu)$ is bounded if and only if $\mu$ is an $\alpha$-Carleson measure. It is natural to ask when $\partial: N_{\alpha}^{p} \rightarrow N^{p}(\mu)$ metrically bounded.

Theorem 1.2. Let $1 \leq p<\infty$ and $\alpha>-1$. Given $\mu \geq 0$, the following conditions are equivalent:

(a) $\mu$ is an $(\alpha+p)$-Carleson measure;

(b) $\partial: N_{\alpha}^{p} \rightarrow N^{p}(\mu)$ is metrically bounded.

Thus $\partial$ distinguishes between the properties "bounded" and "metrically bounded", and in particular $\partial$ is not metrically bounded on any $N_{\alpha}^{p}$. This intuitively tells us that, while area Nevanlinna spaces are large enough to retain all the derivatives of their functions, size of derivatives cannot be controlled well.

In [2] it was shown that for composition operators acting on the Smirnov class $\mathcal{N}^{+}$, the properties of compactness, metric compactness, and a notion of boundedness that we call $\tau$-bounded, are all equivalent. The next theorem shows that the situation is the same for certain operators acting on the area Nevanlinna spaces. In particular, the differentiation operator does not distinguish between the properties of compactness and metric compactness, contrary to the case for boundedness and metric boundedness. For the definition of $\tau$-bounded and the notion of compact $\alpha$-Carleson measures, see Section 2.

Theorem 1.3. Let $1 \leq p<\infty, \alpha>-1$ and let $T$ be any of the operators (i) embedding $\subset$, (ii) integration $\mathcal{I}$, or (iii) differentiation $\partial$. Given $\mu \geq 0$, the following conditions are equivalent:

(a) $\mu$ is a compact $\alpha$-Carleson measure; 
(b) $T: N_{\alpha}^{p} \rightarrow N^{p}(\mu)$ is metrically compact;

(c) $T: N_{\alpha}^{p} \rightarrow N^{p}(\mu)$ is compact;

(d) $T: N_{\alpha}^{p} \rightarrow N^{p}(\mu)$ is $\tau$-bounded.

We note the consequence of Theorem 1.3 that, since $\nu_{\alpha}$ is not a compact $\alpha$ Carleson measure, integration is not a compact operator on $N_{\alpha}^{p}$. This is in contrast to integration on $A_{\alpha}^{p}$, which is well known to be compact. Thus, while differentiation is better behaved on $N_{\alpha}^{p}$ than on $A_{\alpha}^{p}$, integration is not as well behaved.

In Section 2 we briefly review various general notions of boundedness(compactness) and give definitions of metric boundedness(compactness). We also review some background on Carleson measures. In Section 3 we give the proofs of higher order versions of Theorems 1.1, 1.2 and 1.3. The section ends with a discussion of extensions that apply to mappings of $N_{\alpha}^{p}$ into $N^{q}(\mu)$, where $q \geq p$. Then in Section 4 we turn to applications of these results. We first consider composition operators acting between area Nevanlinna spaces and show how our work gives a different approach to a recent characterization by A. Haldimann and J. Jarchow of when such an operator is bounded or compact. Our next application is to Volterra operators and their companion operators. We characterize when these operators are bounded or compact, in various senses, on area Nevanlinna spaces. Our final application concerns the spherical derivative $f^{\sharp}=\left|f^{\prime}\right| /\left(1+|f|^{2}\right)$ of a function $f \in H(D)$. We show that $f^{\sharp}$ naturally occurs in the study of area Nevanlinna spaces, and a consequence of our work is that $(1-|z|) f^{\sharp} \in L^{2}(\nu)$ if and only if $(1-|z|)\left(f^{\prime}\right)^{\sharp} \in L^{2}(\nu)$.

Notation: In the rest of the paper the same letter $C$ will denote various positive constants, unless otherwise specified, which may change at each occurrence. We will often write $X \lesssim Y$ or $Y \gtrsim X$ if $X \leq C Y$ for some positive constant $C$ dependent only on allowed parameters, and $X \approx Y$ if $X \lesssim Y \lesssim X$. Also, we will often use the letter $z$ not only to denote points in $D$, but also to denote the identity function on D.

\section{Prerequisites}

2.1. Bounded(Compact) operators. We first clarify two notions of a bounded set, that are relevant to our work, on a metrizable topological vector space. A subset $A$ of a topological vector space $X$ is called $\tau$-bounded ( $\tau$ for "topology") if, for every neighborhood $V$ of the origin, $A$ is absorbed in $V$, or said differently, $A \subset t V$ for some $t>0$. If the topology of $X$ is given by a metric $d$, there is another natural notion of a bounded set. That is, $A \subset X$ is called d-bounded if $A$ is contained in a metric ball. These two notions of boundedness do not agree in general. In fact no metric ball of an area Nevanlinna space is $\tau$-bounded; see Corollary 3.9 below. For the rest of the paper, the term "bounded" (for a set) will always refer to the $d$-boundedness.

Let $T: X \rightarrow Y$ be a linear operator where $X$ and $Y$ are general topological vector spaces. Various notions of when such an operator is bounded or compact have appeared in the literature. We say that $T$ is bounded if it takes $\tau$-bounded sets to $\tau$-bounded sets (see [19]); $T$ is $\tau$-bounded if it takes some neighborhood of the origin to a $\tau$-bounded set (see [2]); $T$ is compact if it maps some neighborhood of the origin to a relatively compact set. Since relatively compact sets are $\tau$-bounded, compact linear operators are always $\tau$-bounded. It is not hard to see that $\tau$-bounded linear operators are always continuous and conversely if $Y$ possesses a $\tau$-bounded 
neighborhood of the origin. In addition, continuous linear operators are always bounded and conversely if $X$ is metrizable; see [19, Theorem 1.32].

Now, we further assume that topologies of $X$ and $Y$ are given by translationinvariant metrics $d_{X}$ and $d_{Y}$, respectively. We introduce additional notions of boundedness and compactness which are motivated by those given in [2]. We say that $T$ is metrically bounded if there exists some constant $C>0$ such that

$$
d_{Y}(0, T x) \leq C d_{X}(0, x)
$$

for all $x \in X$. Thus $T$ is metrically bounded if and only if $T$ maps every metric ball $B$ in $X$ into another in $Y$ with radius a fixed multiple of the radius of $B$. Also, $T$ is said to be metrically compact if it takes every metric ball in $X$ into a relatively compact set in $Y$. Due to the metric topology of $Y$, the operator $T$ will be metrically compact if and only if the image of every bounded sequence in $X$ has a subsequence that converges in $Y$. We remark that if the metrics satisfy $d(t x, t y)=|t| d(x, y)$, then metric boundedness(compactness) coincide with the standard definitions, mentioned above, of bounded(compact) linear operators on a topological vector space.

Clearly, metric boundedness and metric compactness imply continuity and compactness, respectively. However, a metrically compact linear operator may not be metrically bounded. In fact, as consequences of Theorem 1.3, Theorem 1.2 and the Carleson characterizations for Bergman spaces stated below, we have $\partial^{k}: N_{\alpha}^{p} \rightarrow N_{\beta}^{p}$ is metrically bounded if and only if $\beta \geq \alpha+p k$, while it is metrically compact if and only if $\beta>\alpha$.

2.2. Carleson measures. We now recall the well-known notion of Carleson measures for the weighted Bergman spaces $A_{\alpha}^{p}$. Here, the parameter range of $p$ is restricted to $1 \leq p<\infty$ to be consistent with that for area Nevanlinna spaces, while all the Carleson (type) characterizations mentioned for $A_{\alpha}^{p}$ in this subsection still holds for $0<p<1$.

We say that $\mu \geq 0$ is a Carleson measure for $A_{\alpha}^{p}$ if there exists some constant $C$ such that

$$
\int_{D}|f|^{p} d \mu \leq C \int_{D}|f|^{p} d \nu_{\alpha}
$$

for $f \in A_{\alpha}^{p}$. That is, $\mu$ is a Carleson measure for $A_{\alpha}^{p}$ if and only if the embedding $A_{\alpha}^{p} \subset L^{p}(\mu)$ is continuous, or equivalently by the Closed Graph Theorem, if and only if $A_{\alpha}^{p} \subset L^{p}(\mu)$. If, in addition, the embedding $A_{\alpha}^{p} \subset L^{p}(\mu)$ is compact, then $\mu$ is said to be a compact Carleson measure for $A_{\alpha}^{p}$. Various characterizations for Carleson measures for $A_{\alpha}^{p}$ are well known. Among those characterizations, we recall below the characterization in terms of averaging functions over pseudohyperbolic disks.

The pseudohyperbolic distance $\rho(z, w)$ between $z$ and $w$ in $D$ is given by

$$
\rho(z, w)=\left|\frac{z-w}{1-\bar{z} w}\right| .
$$

For $0<r<1$ we let $E_{r}(z)$ denote the pseudohyperbolic disk with radius $r$ and center $z$. Note that $E_{r}(z)$ is a Euclidean disk with

$$
(\text { center })=\frac{\left(1-r^{2}\right) z}{1-|z|^{2} r^{2}} \quad \text { and } \quad(\text { radius })=\frac{\left(1-|z|^{2}\right) r}{1-|z|^{2} r^{2}}
$$


We recall a well-known estimate which is helpful in dealing with estimates involving pseudohyperbolic disks (see, for example, [21, Lemma 2.27]): Given $0<r<1$ and $s$ real, there exists a constant $C=C(r, s)$ such that

$$
\left|\left(\frac{1-a \bar{z}}{1-a \bar{w}}\right)^{s}-1\right| \leq C \rho(z, w)
$$

for all $a, z, w \in D$ with $\rho(z, w)<r$. We will frequently and tacitly use the following consequences for $z, w \in D$ with $\rho(z, w)<r$ :

(1) $\frac{1-|z|^{2}}{|1-z \bar{w}|} \approx \frac{1-|z|^{2}}{1-|w|^{2}} \approx 1$.

(2) $\Re\left(\frac{1-|z|^{2}}{1-z \bar{w}}\right)^{s} \approx 1 \quad$ (if $r=r(s)$ is sufficiently small).

(3) $\nu_{\alpha}\left[E_{r}(z)\right] \approx(1-|z|)^{2+\alpha}$.

Given $\mu \geq 0, \alpha>-1$, and $0<r<1$, we let $\widehat{\mu}_{\alpha, r}$ denote the averaging function of $\mu$ defined by

$$
\widehat{\mu}_{\alpha, r}(z)=\frac{\mu\left[E_{r}(z)\right]}{\nu_{\alpha}\left[E_{r}(z)\right]} .
$$

A basic inequality satisfied by these averaging functions is the following:

$$
\int_{D} h d \mu \leq C \int_{D} h \widehat{\mu}_{\alpha, r} d \nu_{\alpha}
$$

for all functions $h \geq 0$ subharmonic on $D$ and for some constant $C=C(\alpha, r)$. In order to see this, note that subharmonicity yields

$$
h(z) \leq C(\alpha, r) \int_{E_{r}(z)} \frac{h(w)}{\left(1-|w|^{2}\right)^{2+\alpha}} d \nu_{\alpha}(w)
$$

for $z \in D$. Now, integrating both sides against the measure $d \mu(z)$ and then changing the order of integrations, one obtains (2.1). The inequality (2.1) tells us that if $\widehat{\mu}_{\alpha, r}$ is bounded, then the embedding $A_{\alpha}^{p} \subset L^{p}(\mu)$ is bounded. Also, it turns out that the converse is also true by considering suitable test functions. Furthermore, its compact version is also available. So, we have the following characterization for each $r$ (see, for example, [4, Theorem 2.36] or [21, Theorems 2.15 and 2.16] for details):

- $\mu$ is a Carleson measure for $A_{\alpha}^{p} \Longleftrightarrow \widehat{\mu}_{\alpha, r} \in L^{\infty}(D)$.

- $\mu$ is a compact Carleson measure for $A_{\alpha}^{p} \Longleftrightarrow \widehat{\mu}_{\alpha, r}(z) \rightarrow 0$ as $|z| \rightarrow 1$.

Note that these characterizations are independent of parameters $p$ and $r$. Hence we will sometimes simplify the notation by writing $\widehat{\mu}_{\alpha}$ in place of $\widehat{\mu}_{\alpha, r}$. Also we will sometimes simply say "(compact) $\alpha$-Carleson measure" instead of "(compact) Carleson measure for $A_{\alpha}^{p}$ ".

Carleson type characterizations for differentiation are also well known. Given an integer $k \geq 1$, characterizations are as follows (for each $r$ ):

- $\partial^{k}: A_{\alpha}^{p} \rightarrow L^{p}(\mu)$ is bounded $\Longleftrightarrow \widehat{\mu}_{\alpha+k p, r} \in L^{\infty}(D)$.

- $\partial^{k}: A_{\alpha}^{p} \rightarrow L^{p}(\mu)$ is compact $\Longleftrightarrow \widehat{\mu}_{\alpha+k p, r}(z) \rightarrow 0$ as $|z| \rightarrow 1$.

This was first proved in [13, Theorem 2.2]. Also, see [3, Lemma 2.5] for details (in terms of the so-called Carelson squares) of compactness. We will also sometimes simplify the notation by writing $\widehat{\mu}_{\alpha+k p}$ in place of $\widehat{\mu}_{\alpha+k p, r}$. Later we will use these characterizations without any further reference. 


\section{Carleson Measures}

Recall that in the Introduction we defined $\mu \geq 0$ to be a Carleson measure for $N_{\alpha}^{p}$ if and only if $N_{\alpha}^{p} \subset N^{p}(\mu)$, and since $N_{\alpha}^{p}$ is a metric space this is equivalent to the embedding $N_{\alpha}^{p} \subset N^{p}(\mu)$ being continuous or bounded in the sense of a bounded operator on a topological vector space. Similarly, $\mu$ is said to be a compact Carleson measure for $N_{\alpha}^{p}$ if and only if the embedding $N_{\alpha}^{p} \subset N^{p}(\mu)$ is compact.

Our goal in this section is to obtain characterizations of (compact) Carleson measures for area Nevanlinna spaces in terms of averaging functions. Our characterizations reveal that Carleson measures for area Nevanlinna spaces and Bergman spaces are exactly the same, which is not too surprising, because area Nevanlinna spaces can be viewed as the limiting spaces of the Bergman spaces. In addition, our characterizations reveal some facts about metric boundedness of integration and a similar property of differentiation that may not be expected.

The following inequalities are useful for the characterization involving differentiation.

Proposition 3.1. Let $1 \leq p<\infty, \alpha>-1,0<r<1$ and $k \geq 1$ be an integer. Then there exist constants $C=C(p, \alpha, r, k)$ such that the following inequalities hold:

(a) $\left\|f^{(k)}\right\|_{N^{p}(\mu)}^{p} \leq C \int_{D}\left\{[\log (1+|f|)]^{p}+\left[\log \left(\frac{2}{1-|z|}\right)\right]^{p}\right\} \widehat{\mu}_{\alpha, r} d \nu_{\alpha}$;

(b) $\left\|f^{(k)}\right\|_{N^{p}(\mu)}^{p} \leq C \int_{D}[\log (1+|f|)]^{p} \widehat{\mu}_{\alpha+k p, r} d \nu_{\alpha}$

for all $\mu \geq 0$ and $f \in H(D)$.

Proof. Let $\mu \geq 0$ and $f \in H(D)$. Let $z \in D$. Put $\omega_{p}(x)=[\log (1+x)]^{p}$ for simplicity. By subharmonicity we have

$$
|f(a)| \leq \omega_{p}^{-1}\left\{C \int_{E_{r / 2}(a)} \frac{\omega_{p}(|f(w)|)}{(1-|w|)^{2+\alpha}} d \nu_{\alpha}(w)\right\}, \quad a \in D
$$

for some constant $C=C(\alpha, r)$. A routine calculation shows that the disk with center $z$ and radius $r(1-|z|) / 2$ is contained in $E_{r / 2}(z)$. Thus, applying Cauchy's estimate on that disk, we have

$$
\left|f^{(k)}(z)\right| \leq \frac{1}{r_{1}^{k}(1-|z|)^{k}} \sup _{a \in E_{r / 2}(z)} \omega_{p}^{-1}\left(C \int_{E_{r / 2}(a)} \frac{\omega_{p}(|f(w)|)}{(1-|w|)^{2+\alpha}} d \nu_{\alpha}(w)\right)
$$

where $r_{1}=r / 2$ and thus

$$
\begin{aligned}
\omega_{p}\left(\left|f^{(k)}(z)\right|\right) & \lesssim \omega_{p}\left(\frac{1}{r_{1}^{k}(1-|z|)^{k}}\right)+\sup _{a \in E_{r / 2}(z)} \int_{E_{r / 2}(a)} \frac{\omega_{p}(|f(w)|)}{(1-|w|)^{2+\alpha}} d \nu_{\alpha}(w) \\
& \lesssim\left[\log \left(\frac{2}{1-|z|}\right)\right]^{p}+\int_{E_{r}(z)} \frac{\omega_{p}(|f(w)|)}{(1-|w|)^{2+\alpha}} d \nu_{\alpha}(w) .
\end{aligned}
$$

Note that the function $[-\log (1-|z|)]^{p}$ is subharmonic. Thus, integrating against the measure $d \mu(z)$ and then changing the order of integrations, we conclude (a) by (2.1).

The proof above showed that

$$
\omega_{p}\left(r_{1}^{k}(1-|z|)^{k}\left|f^{(k)}(z)\right|\right) \lesssim \int_{E_{r}(z)} \frac{\omega_{p}(|f(w)|)}{(1-|w|)^{2+\alpha}} d \nu_{\alpha}(w) .
$$


Thus, using the elementary inequality $y \log (1+x) \leq \log (1+x y)$ for $x \geq 0$ and $0 \leq y \leq 1$, we obtain

$$
\begin{aligned}
\omega_{p}\left(\left|f^{(k)}(z)\right|\right) & \lesssim \frac{1}{(1-|z|)^{k p}} \int_{E_{r}(z)} \frac{\omega_{p}(|f(w)|)}{(1-|w|)^{2+\alpha}} d \nu_{\alpha}(w) \\
& \approx \int_{E_{r}(z)} \frac{\omega_{p}(|f(w)|)}{(1-|w|)^{k p+2+\alpha}} d \nu_{\alpha}(w) .
\end{aligned}
$$

Having this estimate, one may now repeat the argument above and conclude (b). The proof is complete.

A preliminary result is needed for the characterization involving integration. Note that we have

$$
\left|\mathcal{I}^{k} f(z)\right| \leq \sup _{0 \leq t \leq 1}|f(t z)|
$$

for $f \in H(D)$ and integers $k \geq 1$. By this we are led to consider an area analogue of the well-known radial maximal function. Given a measurable function $h$ on $D$, we define $\mathcal{M} h$ by

$$
\mathcal{M} h(z)=\sup _{0 \leq t \leq 1}|h(t z)| .
$$

For $1<p \leq \infty$, but not for $p=1$, the Hardy-Littlewood maximal theorem ([5, Theorem 1.8]) implies that this maximal operator, when restricted to nonnegative subharmonic functions, is bounded on $L^{p}\left(\nu_{\alpha}\right)$. We show this extends to the end point case $p=1$ in Theorem 3.2 (with $\mu=\nu_{\alpha}$ ) below.

Given $z \in D$ and $0<r<1$, let $P_{r}(z) \subset \partial D$ be the radial projection of $E_{r}(z)$ and $S_{r}(z)$ be the interior of the convex hull generated by $P_{r}(z)$ and $E_{r}(z)$. Also, given $\mu \geq 0$, let

$$
\widetilde{\mu}_{\alpha}(z)=\widetilde{\mu}_{\alpha, r}(z)=\frac{\mu\left[S_{r}(z)\right]}{\nu_{\alpha}\left[S_{r}(z)\right]} .
$$

Theorem 3.2. Given $\alpha>-1$ and $0<r<1$, there exists a constant $C=C(\alpha, r)$ such that

$$
\int_{D} \mathcal{M} h d \mu \leq C \int_{D} h \widetilde{\mu}_{\alpha, r} d \nu_{\alpha}
$$

for all functions $h \geq 0$ subharmonic on $D$ and $\mu \geq 0$.

Proof. Fix $\alpha>-1$ and $0<r<1$. Let $h \geq 0$ be a subharmonic function on $D$. Let $z \in D$. We have by $(2.2)$

$$
\begin{aligned}
\mathcal{M} h(z) & \lesssim \sup _{0 \leq t \leq 1} \int_{E_{r}(t z)} \frac{h(w)}{\left(1-|w|^{2}\right)^{2+\alpha}} d \nu_{\alpha}(w) \\
& \leq \int_{D}\left\{\sup _{0 \leq t \leq 1} \chi_{E_{r}(t z)}(w)\right\} \frac{h(w)}{\left(1-|w|^{2}\right)^{2+\alpha}} d \nu_{\alpha}(w) \\
& =\int_{D}\left\{\sup _{0 \leq t \leq 1} \chi_{E_{r}(w)}(t z)\right\} \frac{h(w)}{\left(1-|w|^{2}\right)^{2+\alpha}} d \nu_{\alpha}(w) .
\end{aligned}
$$

Here, $\chi_{E}$ denotes the characteristic function of $E \subset D$. Note that we have

$$
\sup _{0 \leq t \leq 1} \chi_{E_{r}(w)}(t z)=\chi_{S_{r}(w)}(z)
$$


Therefore, given $\mu \geq 0$, integrating against the measure $d \mu(z)$ and then changing the order of integrations, we have

$$
\int_{D} \mathcal{M} h(z) d \mu(z) \leq C \int_{D} h(w) \frac{\mu\left[S_{r}(w)\right]}{\left(1-|w|^{2}\right)^{2+\alpha}} d \nu_{\alpha}(w)
$$

for some constant $C=C(\alpha, r)$.

It remains to estimate the size the sets $S_{r}(w)$ :

$$
C^{-1}(1-|w|)^{2+\alpha} \leq \nu_{\alpha}\left[S_{r}(w)\right] \leq C(1-|w|)^{2+\alpha}
$$

for some constants $C=C(\alpha, r)$. Note that $E_{r}(w) \subset S_{r}(w)$, which yields the lower estimate of (3.3). In order to see the upper estimate of (3.3), note that we have

and thus

$$
P_{r}(w)=\left\{\zeta \in \partial D: \frac{\left(1-r^{2}\right)|w| \zeta}{1-|w|^{2} r^{2}} \in E_{r}(w)\right\}
$$

$$
s:=\text { length of } P_{r}(w) \approx \frac{\left(1-|w|^{2}\right) r}{1-|w|^{2} r^{2}} \approx(1-|w|) .
$$

Next, for $|w|>r$, note that we have

$$
t:=\inf _{z \in S_{r}(w)}|z|=\frac{\left(1-r^{2}\right)|w|-\left(1-|w|^{2}\right) r}{1-|w|^{2} r^{2}} \geq|w|-\frac{r}{1-r}(1-|w|) .
$$

It follows that

$$
\nu_{\alpha}\left[S_{r}(w)\right] \lesssim s(1-t)^{1+\alpha} \leq \frac{(1-|w|)^{2+\alpha}}{1-r}, \quad|w|>r .
$$

Also, for $|w| \leq r$, we have

$$
\nu_{\alpha}\left[S_{r}(w)\right] \leq 1 \leq\left(\frac{1-|w|}{1-r}\right)^{2+\alpha}, \quad|w| \leq r .
$$

The proof is complete.

Corollary 3.3. Let $1 \leq p<\infty$ and $\alpha>-1$. Then

$$
\partial\left(N_{\alpha}^{p}\right)=N_{\alpha}^{p} \quad \text { and } \quad \mathcal{I}\left(N_{\alpha}^{p}\right)=N_{\alpha, 0}^{p},
$$

where $N_{\alpha, 0}^{p}=\left\{f \in N_{\alpha}^{p}: f(0)=0\right\}$.

Proof. That $\partial\left(N_{\alpha}^{p}\right) \subset N_{\alpha}^{p}$ is immediate from Proposition 3.1(a), while $\mathcal{I}\left(N_{\alpha}^{p}\right) \subset$ $N_{\alpha, 0}^{p}$ follows from (3.2) and Theorem 3.2 (with $\mu=\nu_{\alpha}$ ). The reverse inclusions are then consequences of the usual inverse relationship of $\partial$ and $\mathcal{I}$.

The next lemma will be used to estimate norms in $N_{\alpha}^{p}$. For a proof see [21, Theorem 1.12].

Lemma 3.4. Given $\alpha>-1$ and $s>0$, there exists a constant $C=C(\alpha, s)$ such that

for $z \in D$.

$$
C^{-1} \leq\left(1-|z|^{2}\right)^{s} \int_{D} \frac{d \nu_{\alpha}(w)}{|1-w \bar{z}|^{2+\alpha+s}} \leq C
$$

We are now ready to prove the higher order version of Theorem 1.1. The proof will be competed in two steps. First we prove the following.

Theorem 3.5. Let $1 \leq p<\infty, \alpha>-1$ and $k \geq 1$ be an integer. Given $\mu \geq 0$, the following conditions are equivalent: 
(a) $\mu$ is an $\alpha$-Carleson measure;

(b) The embedding $N_{\alpha}^{p} \subset N^{p}(\mu)$ is metrically bounded;

(c) $\mathcal{I}^{k}: N_{\alpha}^{p} \rightarrow N^{p}(\mu)$ is metrically bounded;

(d) There is a constant $C$ such that

$$
\left\|f^{(k)}\right\|_{N^{p}(\mu)} \leq C\left(1+\|f\|_{N_{\alpha}^{p}}\right), \quad f \in N_{\alpha}^{p} .
$$

Moreover, given $0<r<1$, the quantities

$$
\left\|\widehat{\mu}_{r}\right\|_{\infty}^{1 / p}, \quad \sup _{0 \neq f \in N_{\alpha}^{p}} \frac{\|f\|_{N^{p}(\mu)}}{\|f\|_{N_{\alpha}^{p}}}, \quad \sup _{0 \neq f \in N_{\alpha}^{p}} \frac{\left\|\mathcal{I}^{k} f\right\|_{N^{p}(\mu)}}{\|f\|_{N_{\alpha}^{p}}}, \quad \sup _{f \in N_{\alpha}^{p}} \frac{\left\|f^{(k)}\right\|_{N^{p}(\mu)}}{1+\|f\|_{N_{\alpha}^{p}}}
$$

are all comparable.

Proof. Throughout the proof $r \in(0,1)$ is fixed and sufficiently small, as needed. Let $\mu \geq 0$. First, we assume (a) and prove that (b)-(d) hold. Applying (2.1) to $h=[\log (1+|f|)]^{p}$ with $f \in N_{\alpha}^{p}$, we see that (b) holds with the estimate

$$
\|f\|_{N^{p}(\mu)}^{p} \lesssim\left\|\widehat{\mu}_{\alpha}\right\|_{\infty}\|f\|_{N_{\alpha}^{p}}^{p}, \quad f \in N_{\alpha}^{p}
$$

In order to prove (c), we recall that when one of $\widetilde{\mu}_{\alpha}$ and $\widehat{\mu}_{\alpha}$ belongs to $L^{\infty}(D)$, then both do and their norms are comparable; see [13, p. 89-90]. Using this fact and the inequality $\left[\log \left(1+\left|\mathcal{I}^{k} f\right|\right)\right]^{p} \leq \mathcal{M}[\log (1+|f|)]^{p}$ for $f \in H(D)$, we see that (c) with the estimate

$$
\left\|\mathcal{I}^{k} f\right\|_{N^{p}(\mu)}^{p} \lesssim\left\|\widehat{\mu}_{\alpha}\right\|_{\infty}\|f\|_{N_{\alpha}^{p}}^{p}, \quad f \in N_{\alpha}^{p}
$$

follows from Theorem 3.2. Also, the implication (a) $\Longrightarrow$ (d) with the estimate

$$
\left\|f^{(k)}\right\|_{N^{p}(\mu)}^{p} \lesssim\left\|\widehat{\mu}_{\alpha}\right\|_{\infty}\left(1+\|f\|_{N_{\alpha}^{p}}^{p}\right), \quad f \in N_{\alpha}^{p}
$$

is a consequence of Proposition 3.1(a).

Next, we prove that each of (b)-(d) implies (a) by utilizing suitable test functions. For example, we can take

$$
f_{z}(w)=\exp \left[\frac{1-|z|}{(1-w \bar{z})^{3+\alpha}}\right]^{1 / p}
$$

for $z \in D$ as our test functions. It is easily checked by Lemma 3.4 that $\left\{f_{z}\right\}$ is bounded in $N_{\alpha}^{p}$. Also, note that we have

$$
\log \left|f_{z}(w)\right|=\Re\left[\frac{1-|z|}{(1-w \bar{z})^{3+\alpha}}\right]^{1 / p} \gtrsim(1-|z|)^{-(2+\alpha) / p}
$$

for $w \in E_{r}(z)$. It follows that

$$
\frac{\left\|f_{z}\right\|_{N^{p}(\mu)}^{p}}{\left\|f_{z}\right\|_{N_{\alpha}^{p}}^{p}} \gtrsim\left\|f_{z}\right\|_{N^{p}(\mu)}^{p} \gtrsim \int_{E_{r}(z)} \frac{d \mu(w)}{(1-|z|)^{2+\alpha}} \approx \widehat{\mu}_{\alpha}(z)
$$

for all $z \in D$. This shows that (b) implies (a).

We now prove that (d) implies (a). An elementary induction yields $f_{z}^{(k)}=\bar{z}^{k} g f_{z}$ for some $g \in H(D)$ that is a sum of functions of the form $c(1-|z|)^{\beta}(1-\bar{z} w)^{-\gamma}$, where the parameters $c>0$ and $\gamma>\beta \geq 1 / p$ depend only on $p, \alpha$ and $k$. Thus there exists $s=s(p, \alpha, k)>0$ such that $g(w) \gtrsim(1-|z|)^{-s}$ for $w \in E_{r}(z)$, and by (3.4) we have

$$
\log \left|f_{z}^{(k)}(w)\right| \gtrsim(1-|z|)^{-(2+\alpha) / p}+\log \left(\frac{C}{1-|z|}\right) \gtrsim(1-|z|)^{-(2+\alpha) / p}
$$


for $|z|>1 / 2, w \in E_{r}(z)$ and for some constant $C=C(p, \alpha, k, r)$. Now, assuming (d) and proceeding as above, we get

$$
\frac{\left\|f_{z}^{(k)}\right\|_{N^{p}(\mu)}^{p}}{1+\left\|f_{z}\right\|_{N_{\alpha}^{p}}^{p}} \gtrsim\left\|f_{z}^{(k)}\right\|_{N^{p}(\mu)}^{p} \gtrsim \widehat{\mu}_{\alpha}(z)
$$

for $|z|>1 / 2$. Since $\mu$ is a finite measure, this shows that (d) implies (a).

To show that (c) implies (a), we take the functions $f_{z}^{(k)}$ as our test functions. Note that $\left\{f_{z}^{(k)}\right\}$ is also bounded in $N_{\alpha}^{p}$ by Proposition 3.1(a). Also, note

$$
\mathcal{I}^{k} f_{z}^{(k)}(w)=f_{z}(w)-\sum_{j=0}^{k-1} \frac{f_{z}^{(j)}(0)}{j !} w^{j} .
$$

Clearly, $\left|f_{z}^{(j)}(0)\right| \lesssim 1$ for all $z$ and $j=1, \ldots, k-1$. Thus, for $w \in E_{r}(z)$, we have

$$
\log \left|\mathcal{I}^{k} f_{z}^{(k)}(w)\right| \gtrsim(1-|z|)^{-(2+\alpha) / p}
$$

as $|z| \rightarrow 1$. So, assuming (c) and proceeding as above, we conclude that

$$
\frac{\left\|\mathcal{I}^{k} f_{z}^{(k)}\right\|_{N^{p}(\mu)}^{p}}{\left\|f_{z}^{(k)}\right\|_{N_{\alpha}^{p}}^{p}} \gtrsim\left\|\mathcal{I}^{k} f_{z}^{(k)}\right\|_{N^{p}(\mu)}^{p} \gtrsim \widehat{\mu}_{\alpha}(z)
$$

as $|z| \rightarrow 1$. This yields (a) as before and completes the proof of the first part of the theorem. The second part of the theorem is implicit in the argument given, so the proof is complete.

We now complete the proof of the higher order version of Theorem 1.1 by establishing the equivalence of the remaining statements.

Theorem 3.6. Let $1 \leq p<\infty, \alpha>-1$ and $k \geq 1$ be an integer. Given $\mu \geq 0$, the following conditions are equivalent:
(a) $\mu$ is an $\alpha$-Carleson measure;
(b) $N_{\alpha}^{p} \subset N^{p}(\mu)$;
(c) $\mathcal{I}^{k}\left(N_{\alpha}^{p}\right) \subset N^{p}(\mu)$;
(d) $\partial^{k}\left(N_{\alpha}^{p}\right) \subset N^{p}(\mu)$.

Proof. Let $\mu \geq 0$. That (a) implies each of the other statements is immediate from Theorem 3.5. Also, the equivalence of (b), (c), and (d) is immediate from Corollary 3.3. Fix $r>0$ sufficiently small and assume that (a) does not hold. The proof will be completed by showing that (b) does not hold. Since (a) fails, there exists a sequence $\left\{a_{n}\right\}$ in $D$ such that

$$
\widehat{\mu}_{\alpha}\left(a_{n}\right) \geq 2^{n} \text { and } a_{n} \rightarrow \zeta
$$

for some $\zeta \in \partial D$. We may assume $\zeta=1$. Furthermore, for $M=M(p, \alpha, r)>1$ to be chosen later, we can take a subsequence (still denoted by $\left\{a_{n}\right\}$ ) which satisfies

$$
\left|1-a_{n}\right|<\frac{1-\left|a_{j}\right|}{M^{n}}, \quad j=1, \ldots, n-1
$$

for all $n \geq 2$.

Now define functions $h_{n}$ by

$$
h_{n}(w)=\frac{1-\left|a_{n}\right|}{\left(1-\bar{a}_{n} w\right)^{3+\alpha}}
$$


and put $h=\sum_{n=1}^{\infty}\left(2^{-n} h_{n}\right)^{1 / p}$. Also, put $f=\exp h$. From Lemma 3.4 we get

$$
\|f\|_{N_{a}^{p}} \lesssim 1+\sum_{n=1}^{\infty}\left\|\left(2^{-n} h_{n}\right)^{1 / p}\right\|_{L^{p}\left(\nu_{\alpha}\right)}=1+\sum_{n=1}^{\infty} 2^{-n / p}\left\|h_{n}\right\|_{L^{1}\left(\nu_{\alpha}\right)}^{1 / p}<\infty
$$

so $f \in N_{\alpha}^{p}$. We will show that $f \notin N^{p}(\mu)$, which means that (b) does not hold and thus will complete the proof.

Fix an integer $n \geq 1$ and let $w \in E_{r}\left(a_{n}\right)$. For each $j<n$, we have

$$
\left|h_{j}(w)\right| \leq \frac{1}{\left(1-\left|a_{j}\right|\right)^{2+\alpha}} \leq \frac{1}{\left(M^{n}\left|1-a_{n}\right|\right)^{2+\alpha}} \leq \frac{1}{M^{n}\left(1-\left|a_{n}\right|\right)^{2+\alpha}} .
$$

Also, for each $j>n$, we have

$$
\left|h_{j}(w)\right| \leq \frac{\left|1-a_{j}\right|}{(1-|w|)^{3+\alpha}} \leq \frac{1-\left|a_{n}\right|}{M^{j}(1-|w|)^{3+\alpha}} \lesssim \frac{1}{M^{n}\left(1-\left|a_{n}\right|\right)^{2+\alpha}}
$$

But, for $j=n$, we have

It follows that

$$
\Re h_{n}(w) \approx \frac{1}{\left(1-\left|a_{n}\right|\right)^{2+\alpha}} .
$$

$$
\begin{aligned}
\Re h(w) & \geq \Re\left[\frac{h_{n}(w)}{2^{n}}\right]^{1 / p}-\sum_{j \neq n}\left|\frac{h_{j}(w)}{2^{j}}\right|^{1 / p} \\
& \geq\left[\frac{C_{1}}{2^{n}\left(1-\left|a_{n}\right|\right)^{2+\alpha}}\right]^{1 / p}\left[1-C_{2}\left(\frac{2}{M}\right)^{n / p}\right]
\end{aligned}
$$

for some constants $C_{1}$ and $C_{2}$ depending only on $p, \alpha$ and $r$. Therefore, choosing $M>2\left(2 C_{2}\right)^{p / n}$, we obtain

$$
[\Re h(w)]^{p} \gtrsim \frac{1}{2^{n}\left(1-\left|a_{n}\right|\right)^{2+\alpha}}, \quad w \in E_{r}\left(a_{n}\right)
$$

for all $n$. It follows that

$$
\|f\|_{N^{p}(\mu)}^{p} \geq \sum_{n=1}^{\infty} \int_{E_{r}\left(a_{n}\right)}(\Re h)^{p} d \mu \gtrsim \sum_{n=1}^{\infty} \frac{\widehat{\mu}_{\alpha}\left(a_{n}\right)}{2^{n}} \geq \sum_{n=1}^{\infty} 1=\infty .
$$

Hence $f \notin N^{p}(\mu)$ as required, and the proof is complete.

The next lemma will be used in the proof of Theorem 1.3.

Lemma 3.7. Let $1 \leq p<\infty$ and $\alpha>-1$. For $z \in D$ and $c>0$, define the function $f_{z, c}$ by

$$
f_{z, c}(w)=\exp \left[\frac{c(1-|z|)}{(1-\bar{z} w)^{3+\alpha}}\right]^{1 / p}-1 .
$$

Given an integer $k \geq 0$ and $s>0$, there exists $c=c(p, \alpha, k, s)>0$ such that

$$
\sup _{z \in D}\left\|f_{z, c}^{(k)}\right\|_{N_{\alpha}^{p}}<s .
$$

Proof. First, consider the case $k=0$. We use the inequality $\left|e^{a}-1\right| \leq e^{|a|}-1$, valid for all complex numbers $a$, which is easily verified using the usual series expansion of the exponential function. From this we get

$$
\left[\log \left(1+\left|f_{z, c}(w)\right|\right)\right]^{p} \leq \frac{c(1-|z|)}{|1-\bar{z} w|^{3+\alpha}}
$$


and now $\left\|f_{z, c}\right\|_{N_{\alpha}^{p}}$ can be made uniformly and arbitrarily small as $c \rightarrow 0$ by Lemma 3.4 .

Now, assume $k \geq 1$. By an elementary induction we have $f_{z, c}^{(k)}=\bar{z}^{k}\left(f_{z, c}+1\right) g$ for some $g \in H(D)$ that is a sum of functions of the form $b[c(1-|z|)]^{\beta}(1-\bar{z} w)^{-\gamma}$, where parameters $b$ and $\gamma>\beta \geq 1 / p$ depend only on $p, \alpha$ and $k$. Hence, taking $c \leq 1$, we obtain

$$
\left|f_{z, c}^{(k)}\right| \leq c^{1 / p}\left(1+\left|f_{z, c}\right|\right) \psi
$$

where $\psi(w)=C(1-|w|)^{-N}$ for some constants $C$ and $N$ depending only on $p, \alpha$ and $k$. Thus we have

$$
1+\left|f_{z, c}^{(k)}\right| \leq\left(1+c^{1 / p} \psi\right)\left(1+\left|f_{z, c}\right|\right)
$$

which, in turn, yields

$$
\left\|f_{z, c}^{(k)}\right\|_{N_{\alpha}^{p}} \leq\left\|c^{1 / p} \psi\right\|_{N^{p}\left(\nu_{\alpha}\right)}+\left\|f_{z, c}\right\|_{N_{\alpha}^{p}} .
$$

The second term of the above was already estimated. The first term can be made arbitrarily small as $c \rightarrow 0$ by the Dominated Convergence Theorem. This completes the proof.

We now turn to the proof of the higher order version of Theorem 1.3.

Theorem 3.8. Let $1 \leq p\langle\infty, \alpha\rangle-1$ and $k \geq 1$ be an integer. Let $T$ be any of the operators (i) embedding $\subset$, (ii) integration $\mathcal{I}^{k}$, or (iii) differentiation $\partial^{k}$. Given $\mu \geq 0$, the following conditions are equivalent:
(a) $\mu$ is a compact $\alpha$-Carleson measure;
(b) $T: N_{\alpha}^{p} \rightarrow N^{p}(\mu)$ is metrically compact;
(c) $T: N_{\alpha}^{p} \rightarrow N^{p}(\mu)$ is compact;
(d) $T: N_{\alpha}^{p} \rightarrow N^{p}(\mu)$ is $\tau$-bounded.

Proof. Let $\mu \geq 0$. Fix $r \in(0,1)$, sufficiently small as needed. It is clear that (b) implies (c) and that (c) implies (d), so it suffices to show that (a) implies (b) and that (d) implies (a).

First, we assume (a) and show (b). With Theorem 3.5 granted, the proof here is an easy modification of the proof of [3, Lemma 2.5] and included here for completeness. Let $\left\{f_{n}\right\}$ be a bounded sequence in $N_{\alpha}^{p}$. Then $\left\{T f_{n}\right\}$ is also a bounded sequence in $N_{\alpha}^{p}$, say of norm at most $M / 2$, by Theorem 3.5 (with $\mu=\nu_{\alpha}$ ). We must show that $\left\{T f_{n}\right\}$ contains a subsequence that converges in $N^{p}(\mu)$. Note that a bounded set in $N_{\alpha}^{p}$ is a normal family by (1.2), and so by re-indexing an appropriate subsequence that converges locally uniformly to some $g \in H(D)$, we may assume that $T f_{n} \rightarrow g$ locally uniformly. Note $g \in N_{\alpha}^{p}$ with $\|g\|_{N_{\alpha}^{p}} \leq M / 2$ by Fatou's lemma. Thus, we have $\left\|T f_{n}-g\right\|_{N_{\alpha}^{p}} \leq M$.

We need to show $T f_{n} \rightarrow g$ in $N^{p}(\mu)$. Let $\epsilon>0$ be given. By assumption there exists some $t \in(0,1)$ sufficiently close to 1 such that $\sup _{|z|>t} \widehat{\mu}_{\alpha}(z) \leq \epsilon$. Corresponding to such $t$, we let $s \in(t, 1)$ be the number satisfying $\cup_{|z| \leq t} E_{r}(z)=s D$. Now, setting $d \tau=\chi_{D \backslash s D} d \mu$, we see that $\widehat{\tau}_{\alpha}(z)=0$ for $|z| \leq t$ and $\widehat{\tau}_{\alpha}(z) \leq \widehat{\mu}_{\alpha}(z) \leq \epsilon$ for $|z|>t$. Thus, $\tau$ is an $\alpha$-Carleson measure with $\left\|\widehat{\tau}_{\alpha}\right\|_{\infty} \leq \epsilon$. Accordingly, we have by Theorem 3.5

$$
\begin{aligned}
\left\|T f_{n}-g\right\|_{N^{p}(\mu)}^{p} & =\left\|\left(T f_{n}-g\right) \chi_{s D}\right\|_{N^{p}(\mu)}^{p}+\left\|T f_{n}-g\right\|_{N^{p}(\tau)}^{p} \\
& \lesssim \mu(D)\left\|\left(T f_{n}-g\right) \chi_{s D}\right\|_{\infty}^{p}+\epsilon M^{p} .
\end{aligned}
$$


Note that the constant suppressed in the above inequality is independent of $n$. Now, since $T f_{n} \rightarrow g$ locally uniformly, the above inequality implies that $T f_{n} \rightarrow g$ in $N^{p}(\mu)$ as required.

Next, we assume (d) and show (a). We will prove the contrapositive, so assume that $\mu$ is not a compact $\alpha$-Carleson measure. We must show that no metric ball in $N_{\alpha}^{p}$ is mapped by $T$ into a $\tau$-bounded set in $N^{p}(\mu)$. Let $s>0$ be given. Since $\mu$ is not a compact $\alpha$-Carleson measure, there is a constant $C_{1}>0$ and a sequence of points $\left\{a_{n}\right\}$ in $D$ such that

$$
\widehat{\mu}_{\alpha}\left(a_{n}\right) \geq C_{1} \text { and }\left|a_{n}\right| \rightarrow 1 .
$$

Corresponding to the sequence $\left\{a_{n}\right\}$, define functions $f_{n}$ defined by

$$
f_{n}(w)=\exp \left[\frac{c\left(1-\left|a_{n}\right|\right)}{\left(1-\bar{a}_{n} w\right)^{3+\alpha}}\right]^{1 / p}-1, \quad n=1,2, \ldots
$$

where $c>0$ is a constant to be chosen in a moment. Put $g_{n}=f_{n}$ if $T$ is the embedding operator, $g_{n}=\mathcal{I}^{k} f_{n}$ if $T=\partial^{k}$, and $g_{n}=\partial^{k} f_{n}$ if $T=\mathcal{I}^{k}$. Using Lemma 3.7 and/or Theorem 3.5 (with $\mu=\nu_{\alpha}$ ), we can pick a number $c>0$ such that functions $g_{n}$ are all contained in $B_{s}$, the metric ball in $N_{\alpha}^{p}$ with center 0 and radius $s$.

Now, we prove that $T B_{s}$ is not $\tau$-bounded in $N^{p}(\mu)$. We only consider the case $T=\mathcal{I}^{k}$; other cases are simpler. As in the proof of Theorem 3.5, we have

$$
\log \left|T g_{n}(w)\right|=\log \left|\mathcal{I}^{k} \partial^{k} f_{n}(w)\right| \gtrsim c^{1 / p}\left(1-\left|a_{n}\right|\right)^{-(2+\alpha) / p}
$$

for $w \in E_{r}\left(a_{n}\right)$ as $\left|a_{n}\right| \rightarrow 1$. Now, given $t>0$, we have

$$
\begin{aligned}
\left\|t^{-1} T g_{n}\right\|_{N^{p}(d \mu)}^{p} & \geq \int_{E_{r}\left(a_{n}\right)}\left[\log \left(1+t^{-1}\left|T g_{n}(w)\right|\right)\right]^{p} d \mu(w) \\
& \gtrsim \mu\left[E_{r}\left(a_{n}\right)\right]\left[c\left(1-\left|a_{n}\right|\right)^{-(2+\alpha)}-|\log t|^{p}\right] \\
& \gtrsim \widehat{\mu}_{\alpha}\left(a_{n}\right)\left[c-\left(1-\left|a_{n}\right|\right)^{(2+\alpha)}|\log t|^{p}\right] \\
& \geq C_{1}\left[c-\left(1-\left|a_{n}\right|\right)^{(2+\alpha)}|\log t|^{p}\right] \\
& \geq \frac{c C_{1}}{2}
\end{aligned}
$$

for $\left|a_{n}\right|$ sufficiently close to 1 . This means that $T B_{s}$ is not absorbed in some neighborhood of the origin in $N^{p}(\mu)$. In other words, $T B_{s}$ is not $\tau$-bounded in $N^{p}(\mu)$, as required. This completes the proof.

Taking $\mu=\nu_{\alpha}$ and $T$ the embedding operator, we get the following corollary.

Corollary 3.9. No metric ball in any area Nevanlinna space is $\tau$-bounded.

Theorem 3.5 implies continuity of differentiation on area Nevanlinna spaces, as mentioned in the Introduction. The same theorem also suggests that differentiation might not be metrically bounded on any area Nevanlinna space. This led us to a Carleson type characterization for metric boundedness of differentiation. Our characterization, Theorem 3.10 below, shows that the derivative analogues of Carleson measures for the area Nevanlinna spaces are also the same as they are for the corresponding Bergman spaces.

We now prove the following higher order version of Theorem 1.2. 
Theorem 3.10. Let $1 \leq p<\infty, \alpha>-1$ and $k \geq 1$ be an integer. Given $\mu \geq 0$, the following conditions are equivalent:

(a) $\mu$ is an $(\alpha+k p)$-Carleson measure;

(b) $\partial^{k}: N_{\alpha}^{p} \rightarrow N^{p}(\mu)$ is metrically bounded.

Moreover, given $0<r<1$, the quantities

$$
\left\|\widehat{\mu}_{\alpha+k p, r}\right\|_{\infty}^{1 / p}, \sup _{0 \neq f \in N_{\alpha}^{p}} \frac{\left\|f^{(k)}\right\|_{N^{p}(\mu)}}{\|f\|_{N_{\alpha}^{p}}}
$$

are comparable.

Proof. Let $\mu \geq 0$. That (a) implies (b) is an immediate consequence of Proposition 3.1(b). We now assume (b) and show (a). Given $z \in D$, let

$$
f_{z}(w)=\frac{(1-|z|)^{k+(3+\alpha) / p}}{(1-\bar{z} w)^{(3+\alpha) / p}} .
$$

By Lemma 3.4 we have

$$
\left\|f_{z}\right\|_{N_{\alpha}^{p}}^{p} \leq\left\|f_{z}\right\|_{A_{\alpha}^{p}}^{p} \approx(1-|z|)^{k p+2+\alpha}
$$

for all $z \in D$. Note $\left|f_{z}^{(k)}(w)\right| \leq C$ for some constant $C=C(p, \alpha, k)$ and thus $\log \left(1+\left|f_{z}^{(k)}(w)\right|\right) \approx\left|f_{z}^{(k)}(w)\right|$ for all $z, w \in D$. In addition, we have $\left|f_{z}^{(k)}(w)\right| \approx|z|^{k}$ for $w \in E_{r}(z)$. Thus we have

$$
\left\|f_{z}^{(k)}\right\|_{N^{p}(\mu)}^{p} \gtrsim \int_{E_{r}(z)}\left|f_{z}^{(k)}(w)\right|^{p} d \mu(w) \approx|z|^{k p} \mu\left[E_{r}(z)\right]
$$

for all $z \in D$. Combining these two estimates, we deduce

$$
\widehat{\mu}_{\alpha+k p}(z) \lesssim \frac{\left\|f_{z}^{(k)}\right\|_{N^{p}(\mu)}^{p}}{\left\|f_{z}\right\|_{N_{\alpha}^{p}}^{p}}
$$

for $|z| \geq 1 / 2$. Since $\mu$ is a finite measure, this yields (a) and completes the proof of the first part of the theorem. The second part of the theorem is implicit in the argument given, so the proof is complete.

The results of this section may easily be extended to apply to mappings of $N_{\alpha}^{p}$ into $N^{q}(\mu)$, where $q \geq p$. We will sketch extension for the embedding operator; details of other extensions are left to the interested reader.

Theorem 3.11. Let $1 \leq p \leq q<\infty$ and $\alpha>-1$. Set $s=(2+\alpha) q / p-2$. Given $\mu \geq 0$, the following conditions are equivalent:
(a) $\mu$ is an $s$-Carleson measure;
(b) $A_{\alpha}^{p} \subset L^{q}(\mu)$;
(c) The embedding $N_{\alpha}^{p} \subset N^{q}(\mu)$ is metrically bounded;
(d) $N_{\alpha}^{p} \subset N^{q}(\mu)$.

Proof. The equivalence of (a) and (b) is well known and due to Luecking; see [13, Theorem 2.2]. Assume $\mu$ is an $s$-Carleson measure and let $f \in N_{\alpha}^{p}$. The growth estimate (1.2) shows that

$$
\|f\|_{N^{q}(\mu)} \leq C\|f\|_{N_{\alpha}^{p}}^{1-p / q}\|f\|_{N^{p}\left((1-|z|)^{\alpha-s} d \mu(z)\right)}^{p / q} .
$$


Since $\mu$ is an $s$-Carleson measure, it is clear that $(1-|z|)^{\alpha-s} d \mu(z)$ is an $\alpha$-Carleson measure, and so

$$
\|f\|_{N^{p}\left((1-|z|)^{\alpha-s} d \mu(z)\right)} \leq C\|f\|_{N_{\alpha}^{p}}
$$

by Theorem 3.5. Combining these estimates completes the proof that (a) implies (c). That (c) implies (d) is immediate, and the proof that (d) implies (a) is accomplished by making obvious modifications to the proof of the corresponding part of Theorem 3.6.

\section{Applications}

In this section we collect some applications of the results obtained above.

4.1. Composition operators. Our first application is to composition operators on area Nevanlinna spaces. Let $\varphi \in H(D)$ be a nonconstant self-map of $D$. Associated with $\varphi$ is the composition operator $C_{\varphi}$ defined by

$$
C_{\varphi} f=f \circ \varphi
$$

for $f \in H(D)$. It is clear that $C_{\varphi}$ takes $H(D)$ into itself. Since $\log (1+|f|)^{p}, p \geq 1$, is subharmonic when $f \in H(D)$, the Littlewood Subordination Principle shows $C_{\varphi}$ is always metrically bounded on $N_{\alpha}^{p}$ with estimates

$$
\left\|C_{\varphi} f\right\|_{N_{\alpha}^{p}} \leq\left(\frac{1+|\varphi(0)|}{1-|\varphi(0)|}\right)^{2 / p}\|f\|_{N_{\alpha}^{p}}
$$

for $f \in N_{\alpha}^{p}$.

Recent work of A. Haldimann, H. Jarchow, and J. Xiao has shown that the action of $C_{\varphi}$ between area Nevanlinna spaces is closely related to its action between corresponding Bergman spaces.

Theorem 4.1. ([7]; see also [11] and [20].) Let $1 \leq p \leq q<\infty$ and let $\varphi \in H(D)$ be a self-map of $D$. Then $C_{\varphi}: N_{\alpha}^{p} \rightarrow N_{\beta}^{q}$ is (metrically) bounded[compact] if and only if $C_{\varphi}: A_{\alpha}^{p} \rightarrow A_{\beta}^{q}$ is bounded[compact].

This theorem is easily derived from our work on Carleson measures. We just give the argument for $C_{\varphi}$ bounded, the argument for $C_{\varphi}$ compact being similar.

A change of variables formula from measure theory involving the pullback measure defined by $\nu_{\beta} \circ \varphi^{-1}(E)=\nu_{\beta}\left(\varphi^{-1}(E)\right)$ shows that

$$
\|f \circ \varphi\|_{N_{\beta}^{q}}=\|f\|_{N^{q}\left(\nu_{\beta} \circ \varphi^{-1}\right)} \quad \text { and } \quad\|f \circ \varphi\|_{A_{\beta}^{q}}=\|f\|_{L^{q}\left(\nu_{\beta} \circ \varphi^{-1}\right)} .
$$

Thus, by Theorem 3.11, $C_{\varphi}: N_{\alpha}^{p} \rightarrow N_{\beta}^{q}$ is (metrically) bounded if and only if $\nu_{\beta} \circ \varphi^{-1}$ is an $[(2+\alpha) q / p-2]$-Carleson measure, which, again by Theorem 3.11, is also equivalent to $C_{\varphi}: A_{\alpha}^{p} \rightarrow A_{\beta}^{q}$ being bounded.

4.2. Volterra operators. Next, we characterize various notions of boundedness or compactness of Volterra operators and their companion operators. Given $g \in H(D)$, the Volterra operator $J_{g}$ and its companion operator $I_{g}$ with symbol $g$ are the operators defined by

$$
J_{g} f(z)=\int_{0}^{z} f(\zeta) g^{\prime}(\zeta) d \zeta
$$


and

$$
I_{g} f(z)=\int_{0}^{z} f^{\prime}(\zeta) g(\zeta) d \zeta
$$

for $z \in D$ and $f \in H(D)$. Note that $J_{z}$ is simply the integration operator $\mathcal{I}$ and $J_{g}$ with $g(z)=\log (1-z)^{-1}$ is the classical Cèsaro operator(see [10]). Also, note that we have

$$
J_{g} f+I_{g} f=M_{g} f-f(0) g(0)
$$

where $M_{g}$ denotes the multiplication operator with symbol $g$.

Given a subspace $X \subset H(D)$, let

$$
J[X]=\left\{g \in H(D): J_{g} X \subset X\right\} .
$$

Also, we define $I[X]$ and $M[X]$ similarly. The symbol class $M[X]$ has been extensively studied for various Banach spaces $X$. In case $X=H^{2}$, the Hardy space, Pommerenke [18] first proved $J\left[H^{2}\right]=B M O A$ and initiated the extensive study of describing $J[X]$ or $I[X]$, as well as their compact analogues, for various Banach spaces $X$, including (weighted) Bergman spaces, (little) Bloch space, BMOA and $V M O A$. See [12] and references therein. Recently, Nakazi [15] has studied the case where $X$ is a general algebra which is not necessarily a Banach space. Clearly, we have $M[X]=X$ for an algebra $X$ containing constants. In case $X=\mathcal{N}^{1}$, recall that $z \notin J\left[\mathcal{N}^{1}\right]$ as mentioned in the Introduction and hence $z \notin I\left[\mathcal{N}^{1}\right]$ by (4.1). An open problem in this direction is to prove or disprove $J\left[\mathcal{N}^{1}\right]=I\left[\mathcal{N}^{1}\right]=\mathbf{C}$; see [15].

Here, we consider the case of area Nevanlinna spaces. Note that $g \in J\left[N_{\alpha}^{p}\right]$ if and only if $J_{g}$ is continuous on $N_{\alpha}^{p}$ by the Closed Graph Theorem and the same is also true for $I\left[N_{\alpha}^{p}\right]$ and $M\left[N_{\alpha}^{p}\right]$. To study analogues for metric boundedness, compactness, etc., we introduce symbol classes as follows:

$$
\begin{aligned}
J_{\mathrm{mb}}\left[N_{\alpha}^{p}\right]: & =\left\{g \in H(D): J_{g} \text { is metrically bounded on } N_{\alpha}^{p}\right\} ; \\
J_{\mathrm{mc}}\left[N_{\alpha}^{p}\right]: & =\left\{g \in H(D): J_{g} \text { is metrically compact on } N_{\alpha}^{p}\right\} ; \\
J_{\mathrm{c}}\left[N_{\alpha}^{p}\right]: & =\left\{g \in H(D): J_{g} \text { is compact on } N_{\alpha}^{p}\right\} ; \\
J_{\tau \mathrm{b}}\left[N_{\alpha}^{p}\right] & :=\left\{g \in H(D): J_{g} \text { is } \tau \text {-bounded on } N_{\alpha}^{p}\right\} .
\end{aligned}
$$

The symbol classes $I_{\mathrm{mb}}\left[N_{\alpha}^{p}\right], M_{\mathrm{mb}}\left[N_{\alpha}^{p}\right]$, etc., are defined similarly.

For symbol classes except for metric boundedness, we have complete characterizations, as given in the next theorem.

Theorem 4.2. Let $1 \leq p<\infty$ and $\alpha>-1$. Then the following statements hold:

(a) $M\left[N_{\alpha}^{p}\right]=J\left[N_{\alpha}^{p}\right]=I\left[N_{\alpha}^{p}\right]=N_{\alpha}^{p}$;

(b) $M_{\mathrm{mc}}\left[N_{\alpha}^{p}\right]=I_{\mathrm{mc}}\left[N_{\alpha}^{p}\right]=\{0\}$ and $J_{\mathrm{mc}}\left[N_{\alpha}^{p}\right]=\mathbf{C}$;

(c) $M_{\mathrm{c}}\left[N_{\alpha}^{p}\right]=I_{\mathrm{c}}\left[N_{\alpha}^{p}\right]=\{0\}$ and $J_{\mathrm{c}}\left[N_{\alpha}^{p}\right]=\mathbf{C}$;

(d) $M_{\tau \mathrm{b}}\left[N_{\alpha}^{p}\right]=I_{\tau \mathrm{b}}\left[N_{\alpha}^{p}\right]=\{0\}$ and $J_{\tau \mathrm{b}}\left[N_{\alpha}^{p}\right]=\mathbf{C}$.

Proof. As mentioned above, we have $M\left[N_{\alpha}^{p}\right]=N_{\alpha}^{p}$, because $N_{\alpha}^{p}$ is an algebra containing constants. Note that $J_{g}=\mathcal{I} M_{g^{\prime}}$ and $I_{g}=\mathcal{I} M_{g} \partial$ for $g \in H(D)$. Also, note that $\partial J_{g} 1=g^{\prime}$ and $\partial I_{g} z=g$ for $g \in H(D)$. These observations, together with Corollary 3.3, yield $J\left[N_{\alpha}^{p}\right]=I\left[N_{\alpha}^{p}\right]=N_{\alpha}^{p}$ and finish the proof of (a).

Since a metrically compact operator is compact, and a compact operator is $\tau$ bounded, we have $M_{\mathrm{mc}}\left[N_{\alpha}^{p}\right] \subset M_{\mathrm{c}}\left[N_{\alpha}^{p}\right] \subset M_{\tau \mathrm{b}}\left[N_{\alpha}^{p}\right]$, with analogous containments for the operators $I$ and $J$. Thus (b) and (c) will follow once we prove (d). To 
this end, let $g \in M_{\tau \mathrm{b}}\left[N_{\alpha}^{p}\right]$. Assume $g$ is not identically 0 . Then there exist some $\epsilon \in(0,1)$ and a sequence $\left\{a_{n}\right\}$ of points in $D$ such that

$$
\left|g\left(a_{n}\right)\right| \geq \epsilon>0 \quad \text { and } \quad\left|a_{n}\right| \rightarrow 1
$$

as $n \rightarrow \infty$. Let $s>0$. By Lemma 3.7 we can choose $c>0$ so that the functions

$$
f_{n}(w)=\exp \left[\frac{c\left(1-\left|a_{n}\right|^{2}\right)}{\left(1-\bar{a}_{n} w\right)^{3+\alpha}}\right]^{1 / p}-1
$$

all belong to $B_{s}$, the metric ball in $N_{\alpha}^{p}$ with center 0 radius $s$. Let $t>1$. Using elementary inequality $\log (1+x y) \geq y \log (1+x)$ for $x \geq 0$ and $0 \leq y \leq 1$, we have by (1.2) that

$$
\begin{aligned}
\left\|t^{-1} g f_{n}\right\|_{N_{\alpha}^{p}} & \geq\left(1-\left|a_{n}\right|\right)^{(2+\alpha) / p} \log \left(1+t^{-1}\left|g\left(a_{n}\right) f_{n}\left(a_{n}\right)\right|\right) \\
& \geq \epsilon\left(1-\left|a_{n}\right|\right)^{(2+\alpha) / p} \log \left[1+t^{-1}\left|f_{n}\left(a_{n}\right)\right|\right] \\
& \geq \epsilon\left(1-\left|a_{n}\right|\right)^{(2+\alpha) / p}\left[c^{1 / p}\left(1-\left|a_{n}\right|^{2}\right)^{-(2+\alpha) / p}-\log t\right] \\
& >\epsilon c^{1 / p} 2^{-1-(2+\alpha) / p}
\end{aligned}
$$

for all $n$ sufficiently large. Thus $\left\{M_{g} f_{n}\right\}$ is not contained in $t B_{R}$, where $R$ is some constant $C=C(p, \alpha)$ times the constant appearing at the end of the display above. Since this holds for all $t>1$, we conclude that $\left\{M_{g} f_{n}\right\}$ and hence $M_{g} B_{s}$ is not $\tau$-bounded. Since $s>0$ was arbitrary, this contradicts the assumption that $M_{g}$ is $\tau$-bounded. Thus $g=0$, and we conclude that $M_{\tau \mathrm{b}}\left[N_{\alpha}^{p}\right]=\{0\}$.

Note $M_{g}=\partial I_{g} \mathcal{I}$. Since $\partial$ is continuous and hence bounded on $N_{\alpha}^{p}$, and $\mathcal{I}$ is metrically bounded on $N_{\alpha}^{p}$ by Theorem 3.5, it follows that $I_{\tau \mathrm{b}}\left[N_{\alpha}^{p}\right] \subset M_{\tau \mathrm{b}}\left[N_{\alpha}^{p}\right]=$ $\{0\}$. By a similar argument using the relation $M_{g^{\prime}}=\partial J_{g}$, we get $J_{\tau \mathrm{b}}\left[N_{\alpha}^{p}\right]=\mathbf{C}$. The proof is complete.

In what follows $H^{\infty}$ denotes the algebra of all bounded holomorphic functions on $D$ and $H_{1}^{\infty}$ denotes the algebra of all functions $f \in H^{\infty}$ such that $f^{\prime} \in H^{\infty}$. Also, $\mathcal{B}$ denotes the well-known Bloch space consisting of all functions $f \in H(D)$ such that $\left(1-|z|^{2}\right)\left|f^{\prime}(z)\right|$ is bounded on $D$.

We have the following results for metrically bounded symbol classes. Characterizations for $J_{\mathrm{mb}}\left[N_{\alpha}^{p}\right]$ and $I_{\mathrm{mb}}\left[N_{\alpha}^{p}\right]$ seem to be more subtle and we do not know what they are exactly. The main difficulty is caused by the fact that the differentiation operator $\partial$, involved in the operators $J_{g}$ and $I_{g}$, is not metrically bounded.

Proposition 4.3. Let $1 \leq p<\infty$ and $\alpha>-1$. Then the following statements hold:

(a) $M_{\mathrm{mb}}\left[N_{\alpha}^{p}\right]=H^{\infty}$;

(b) $H_{1}^{\infty} \subset J_{\mathrm{mb}}\left[N_{\alpha}^{p}\right] \subset \mathcal{B}$;

(c) $H_{1}^{\infty} \subset I_{\mathrm{mb}}\left[N_{\alpha}^{p}\right] \subset H^{\infty}$.

Proof. We first prove (a). The containment $H^{\infty} \subset M_{\mathrm{mb}}\left[N_{\alpha}^{p}\right]$ is clear by the elementary inequality $\log (1+x y) \leq \max \{1, y\} \log (1+x)$ for $x, y \geq 0$. Let $g \in H(D)$ and assume $g \in M_{\mathrm{mb}}\left[N_{\alpha}^{p}\right]$. Given $z \in D$, let

$$
f_{z}(w)=\left(\frac{1-|z|^{2}}{1-\bar{z} w}\right)^{\beta / p}
$$


for $z \in D$ where $\beta$ is chosen so that $\beta>2+\alpha+p$. Note $f_{z}(z)=1$. We have by (1.2) and the metric boundedness of $M_{g}$

$$
\log (1+|g(z)|)=\log \left(1+\left|g(z) f_{z}(z)\right|\right) \lesssim\left\|M_{g}\right\|\left\|f_{z}\right\|_{N_{\alpha}^{p}}(1-|z|)^{-(2+\alpha) / p}
$$

for all $z \in D$ where $\left\|M_{g}\right\|$ is the "norm" of $M_{g}$. Meanwhile, we have by Lemma 3.4

$$
\left\|f_{z}\right\|_{N_{\alpha}^{p}}^{p} \leq \int_{D}\left|f_{z}(w)\right|^{p} d \nu_{\alpha}(w) \approx\left(1-|z|^{2}\right)^{2+\alpha} .
$$

Combining these estimates, we see $g \in H^{\infty}$ and thus conclude (a).

Next, assume $g \in J_{\mathrm{mb}}\left[N_{\alpha}^{p}\right]$. Fix $r \in(0,1 / 2)$. Then we have by (3.1) and (4.2)

$$
\begin{aligned}
\log \left[1+r(1-|z|)\left|g^{\prime}(z)\right|\right] & =\log \left[1+r(1-|z|)\left|\left(J_{g} f_{z}\right)^{\prime}(z)\right|\right] \\
& \lesssim\left\|J_{g}\right\|\left\|f_{z}\right\|_{N_{\alpha}^{p}}(1-|z|)^{-(2+\alpha) / p} \\
& \lesssim\left\|J_{g}\right\|
\end{aligned}
$$

for all $z \in D$. This yields $g \in \mathcal{B}$. Thus $J_{\mathrm{mb}}\left[N_{\alpha}^{p}\right] \subset \mathcal{B}$. On the other hand, since $J_{g}=\mathcal{I} M_{g^{\prime}}$ and $\mathcal{I}: N_{\alpha}^{p} \rightarrow N_{\alpha}^{p}$ is metrically bounded by Theorem 3.5, we see $H_{1}^{\infty} \subset J_{\mathrm{mb}}\left[N_{\alpha}^{p}\right]$, completing the proof of (b).

Finally, assume $g \in I_{\mathrm{mb}}\left[N_{\alpha}^{p}\right]$. A similar argument yields

$$
\begin{aligned}
\log [1+r(1-|z|)|g(z)|] & =\log \left[1+r(1-|z|)\left|g(z) f_{z}(z)\right|\right] \\
& =\log \left[1+r(1-|z|)\left|\left(I_{g} \mathcal{I} f_{z}\right)^{\prime}(z)\right|\right] \\
& \lesssim\left\|I_{g}\right\|\left\|\mathcal{I} f_{z}\right\|_{N_{\alpha}^{p}}(1-|z|)^{-(2+\alpha) / p}
\end{aligned}
$$

Meanwhile, since

$$
\mathcal{I} f_{z}(w)=\frac{p}{\beta-p} \cdot \frac{1-|z|^{2}}{\bar{z}}\left\{\left(\frac{1-|z|^{2}}{1-\bar{z} w}\right)^{\beta / p-1}-\left(1-|z|^{2}\right)^{\beta / p-1}\right\},
$$

we have by Lemma 3.4

$$
\left\|\mathcal{I} f_{z}\right\|_{N_{\alpha}^{p}}^{p} \lesssim(1-|z|)^{\beta} \int_{D} \frac{d \nu_{\alpha}(w)}{|1-\bar{z} w|^{\beta-p}}+\left(1-|z|^{2}\right)^{\beta} \approx\left(1-|z|^{2}\right)^{2+\alpha+p}
$$

for $|z| \geq 1 / 2$. Combining these estimates, we obtain

$$
(1-|z|) \log (1+r|g(z)|) \leq \log [1+r(1-|z|)|g(z)|] \lesssim(1-|z|)
$$

for $|z| \geq 1 / 2$. This implies $g \in H^{\infty}$. Thus $I_{\mathrm{mb}}\left[N_{\alpha}^{p}\right] \subset H^{\infty}$. On the other hand, since $J_{\mathrm{mb}}\left[N_{\alpha}^{p}\right] \cap M_{\mathrm{mb}}\left[N_{\alpha}^{p}\right] \subset I_{\mathrm{mb}}\left[N_{\alpha}^{p}\right]$ by (4.1), we have $H_{1}^{\infty} \subset I_{\mathrm{mb}}\left[N_{\alpha}^{p}\right]$ by (a) and (b), completing the proof of (c). The proof is complete.

Remarks. (1) As a consequence of (4.1) and Proposition 4.3, we have $I_{\mathrm{mb}}\left[N_{\alpha}^{p}\right]=$ $J_{\mathrm{mb}}\left[N_{\alpha}^{p}\right] \cap H^{\infty}$. Also, note that $I_{f} I_{g}=I_{f g}$. Thus $I_{\mathrm{mb}}\left[N_{\alpha}^{p}\right]$ is an algebra.

(2) As mentioned earlier, we have $J\left[H^{2}\right]=B M O A([18])$. The Bergman space analogue is proved in [1]: $J\left[A_{\alpha}^{p}\right]=\mathcal{B}$ and, moreover, $J_{\mathrm{c}}\left[A_{\alpha}^{p}\right]=\mathcal{B}_{0}$, the little Bloch space. This and Theorem 4.2 reveal quite a different nature of area Nevanlinna spaces and Bergman spaces, as far as the compact symbol classes for Volterra operators are concerned. However, for the other operators, we have exactly the same characterizations on Bergman spaces and area Nevanlinna spaces. That is, we have $M\left[A_{\alpha}^{p}\right]=H^{\infty}$ and $M_{\mathrm{c}}\left[A_{\alpha}^{p}\right]=\{0\}$, as is well known, and also, easily modifying the arguments of [1], one can deduce $I\left[A_{\alpha}^{p}\right]=H^{\infty}$ and $I_{\mathrm{c}}\left[A_{\alpha}^{p}\right]=\{0\}$. 
(3) Given $f \in H(D)$, one can deduce from (3.1) that

$$
\int_{D}\left[\log \left(1+(1-|z|)\left|f^{\prime}\right|\right]^{p} d \nu_{\alpha} \leq C \int_{D}[\log (1+|f-f(0)|)]^{p} d \nu_{\alpha}\right.
$$

For the converse, however, what we've so far is

$$
\int_{D}[\log (1+|f-f(0)|)]^{p} d \nu_{\alpha} \leq C\left[1+\int_{D}\left[\log \left(1+(1-|z|)\left|f^{\prime}\right|\right]^{p} d \nu_{\alpha}\right],\right.
$$

which is easily seen from Theorem 3.5(c) (with $k=1$ and $\mu=\nu_{\alpha}$ ). Attempting to identify the symbol classes in (b) or (c) of Proposition 4.3, we are led to a question whether the additive constant term in (4.3) would be indispensable, which would be stronger than the metric boundedness of $\mathcal{I}$ on $N_{\alpha}^{p}$. Recall $\partial: N_{\alpha}^{p} \rightarrow N_{\alpha+p}^{p}$ is metrically bounded by Theorem 3.10 . That is, we have

$$
\int_{D}\left[\log \left(1+\left|f^{\prime}\right|\right)\right]^{p} d \nu_{\alpha+p} \leq C \int_{D}[\log (1+|f-f(0)|)]^{p} d \nu_{\alpha} .
$$

If the converse inequality of the above were to hold, then the additive constant term in (4.3) could be removed. But, it certainly fails, because $\mathcal{I}\left(N_{\alpha+p}^{p}\right) \not \subset N_{\alpha}^{p}$ by Corollary 3.3 .

4.3. Spherical derivatives. Finally, we deduce some integral behavior of spherical derivatives. Recall that the spherical derivative $f^{\sharp}$ of $f \in H(D)$ is the function on $D$ defined by

$$
f^{\sharp}=\frac{\left|f^{\prime}\right|}{1+|f|^{2}} .
$$

This spherical derivative naturally occurs in the study of the unweighted area Nevanlinna space $N_{0}^{1}$. In order to see that, we need to introduce some notation.

Given $f \in N_{0}^{p}$, let

$$
\|\mid f\|_{N_{0}^{p}}^{p}=\int_{D}\left[\log \left(1+|f|^{2}\right)\right]^{p} d \nu
$$

Note that

$$
\frac{1}{2}\left\|\left|f\left\|\left.\right|_{N_{0}^{p}} ^{p} \leq\right\| f\left\|_{N_{0}^{p}}^{p} \leq \log 2+\right\|\right| f\right\|_{N_{0}^{p}}^{p} .
$$

The advantage of this new norm $\left\||\cdot \||_{N_{0}^{p}}\right.$ lies in the integral representation provided by Proposition 4.4 below.

For $1 \leq p<\infty$ we let

$$
u_{p}(x)=[\log (1+x)]^{p} \quad \text { and } \quad U_{p}(x)=x u_{p}^{\prime \prime}(x)+u_{p}^{\prime}(x), \quad x \geq 0
$$

for simplicity. A routine calculation yields

$$
U_{p}(x)=\frac{p[\log (1+x)]^{p-1}}{(1+x)^{2}}\left\{1+\frac{(p-1) x}{\log (1+x)}\right\}
$$

Note that we have

$$
\Delta\left(u_{p} \circ|f|^{2}\right)=4\left(U_{p} \circ|f|^{2}\right)\left|f^{\prime}\right|^{2}
$$

for $f \in H(D)$ where $\Delta$ denotes the Laplacian.

Proposition 4.4. Let $1 \leq p<\infty$. If $f \in N_{0}^{p}$, then

$$
\|\left.|f|\right|_{N_{0}^{p}} ^{p}=\left(u_{p} \circ|f|^{2}\right)(0)+\int_{D}\left(U_{p} \circ|f|^{2}\right)\left|f^{\prime}\right|^{2}\left(|z|^{2}-1+\log \frac{1}{|z|^{2}}\right) d \nu .
$$


Proof. We first recall the integral identity

$$
\frac{1}{2 \pi} \int_{0}^{2 \pi} h\left(r e^{i \theta}\right) d \theta=h(0)+\frac{1}{2} \int_{r D}(\Delta h) \log \frac{r}{|z|} d \nu
$$

for $h \in C^{2}(D)$ and $0 \leq r<1$. This can be verified by the standard argument using Green's theorem: see, for example, [17]. Let $f \in N_{0}^{p}$. Applying the above identity to the function $h=u_{p} \circ|f|^{2}$, we have by (4.4)

$$
\frac{1}{2 \pi} \int_{0}^{2 \pi}\left(u_{p} \circ|f|^{2}\right)\left(r e^{i \theta}\right) d \theta=\left(u_{p} \circ|f|^{2}\right)(0)+2 \int_{r D}\left(U_{p} \circ|f|^{2}\right)\left|f^{\prime}\right|^{2} \log \frac{r}{|z|} d \nu
$$

for $0 \leq r<1$. Now, integrating both sides above against the measure $2 r d r$ on $[0,1)$ and then applying Fubini's theorem, we obtain

$$
\begin{aligned}
\|\mid f\|_{N_{0}^{p}}^{p} & =\int_{D} u_{p} \circ|f|^{2} d \nu \\
& =\left(u_{p} \circ|f|^{2}\right)(0)+4 \int_{D}\left(U_{p} \circ|f|^{2}\right)\left|f^{\prime}\right|^{2}\left(\int_{|z|}^{1} r \log \frac{r}{|z|} d r\right) d \nu \\
& =\left(u_{p} \circ|f|^{2}\right)(0)+\int_{D}\left(U_{p} \circ|f|^{2}\right)\left|f^{\prime}\right|^{2}\left(|z|^{2}-1+\log \frac{1}{|z|^{2}}\right) d \nu
\end{aligned}
$$

as desired. The proof is complete.

The case $p=1$ of Proposition 4.4 is of particular interest, because it reduces to

$$
\left\|\left.\left|f \|_{N_{0}^{1}}=\log \left(1+|f(0)|^{2}\right)+\int_{D}\right| f^{\sharp}\right|^{2}\left(|z|^{2}-1+\log \frac{1}{|z|^{2}}\right) d \nu .\right.
$$

Note that

$$
|z|^{2}-1+\log \frac{1}{|z|^{2}} \approx\left(1-|z|^{2}\right)^{2}
$$

as $|z| \rightarrow 1$. Thus we have

$$
f \in N_{0}^{1} \Longleftrightarrow f^{\sharp} \in L^{2}\left(\nu_{2}\right)
$$

for $f \in H(D)$. Thus, by Corollary 3.3, we have the following result.

Theorem 4.5. Let $f \in H(D)$. Then $f^{\sharp} \in L^{2}\left(\nu_{2}\right)$ if and only if $\left(f^{\prime}\right)^{\sharp} \in L^{2}\left(\nu_{2}\right)$.

This is, of course, very different from the general integral behavior of ordinary derivatives. For example, it is well known that $f \in H(D)$ belongs to $L^{2}(\nu)$ if and only if $f^{\prime} \in L^{2}\left(\nu_{2}\right)$; see [9, Proposition 1.11].

\section{REFERENCES}

[1] A. Aleman and A. G. Siskakis, Integration operators on Bergman spaces, Indiana Univ. Math. J., 46 (1997), no. 2, 337-356.

[2] J. S. Choa, H. O. Kim and J. H. Shapiro, Compact Composition operators on the Smirnov class, Proc. Am. Math. Soc., 128(1999), 2297-2308.

[3] B. R. Choe, H. Koo and W. Smith, Composition operators acting on holomorphic Sobolev spaces, Trans. Amer. Math. Soc., 355(2003), 2829-2855.

[4] C. Cowen and B. MacCluer, Composition operators on spaces of analytic functions, CRC Press, New York, 1995.

[5] P. Duren, Theory of $H^{p}$ spaces, Academic, New York, 1970.

[6] O. Frostman, Sur les produits de Blaschke, Kungl. Fysiogr. Sällsk. i Lund Förh., 12(1942), no. $15,169-182$.

[7] A. Haldimann and H. Jarchow, Nevanlinna algebras, Studia Math., 147 (2001), 243-268. 
B. CHOE, H. KOO, AND W. SMITH

[8] W. K. Hayman, On the characteristic of functions meromorphic in the unit disk and of their integrals, Acta Math., 112(1964), 181-214.

[9] H. Hedenmalm, B. Korenblum and K. Zhu, Theory of Bergman spaces, Springer, New York, 2000.

[10] Z. Hu, Extended Cèsaro operators on Bergman spaces, J. Math. Anal. Appl., 296(2004), 435-454.

[11] H. Jarchow and J. Xiao, Composition operators between Nevanlinna classes and Bergman spaces with weights, J. Operator Theory, 46(2001), no. 3, suppl., 605-618.

[12] S. Li, Volterra composition operators between weighted Bergman spaces and Bloch type spaces, preprint.

[13] D. Luecking, Forward and Reverse Carleson inequalities for functions in Bergman spaces and their derivatives, Amer. J. Math., 107(1985), 85-111

[14] B. D. MacCluer and J. H. Shapiro, Angular derivatives and comapct composition operators on the Hardy and Bergman spaces, Canad. J. Math., 38(1986), 878-906.

[15] T. Nakazi, Integral operators on a subspace of holomorphic functions on the disc, preprint.

[16] R. Nevanlinna, Le théorème de Picard-Borel et la théorie des fonctions meromorphes, Gauthiers-Villars, Paris, 1929, p.138.

[17] M. Pavlović, Inequalities for the gradient of eigenfunctions of the invariant Laplacian in the unit ball, Indag. Math. (N.S.), 2(1991), 89-98.

[18] Ch. Pommerenke, Schlichte funktionen und analytische funktionen von beschrankenr mittlerer oszillation, Comment. Math. Helv., 52(1977), 591-602.

[19] W. Rudin, Functional Analysis, Second edition, McGraw-Hill, New York, 1991.

[20] J. Xiao, Compact Composition operators on the area-Nevanlinna class, Expo. Math., 17(1999), 255-264.

[21] K. Zhu, Spaces of holomorphic functions in the unit ball, Springer, New York, 2000.

Department of Mathematics, Korea University, Seoul 136-713, Korea

E-mail address: cbr@korea.ac.kr

Department of Mathematics, Korea University, Seoul 136-713, Korea

E-mail address: koohw@korea.ac.kr

Department of Mathematics, University of Hawait, Honolulu, Hawail 96822

E-mail address: wayne@math.hawaii.edu 